\title{
Relationship between iron status markers and insulin resistance: an exploratory study in subjects with excess body weight
}

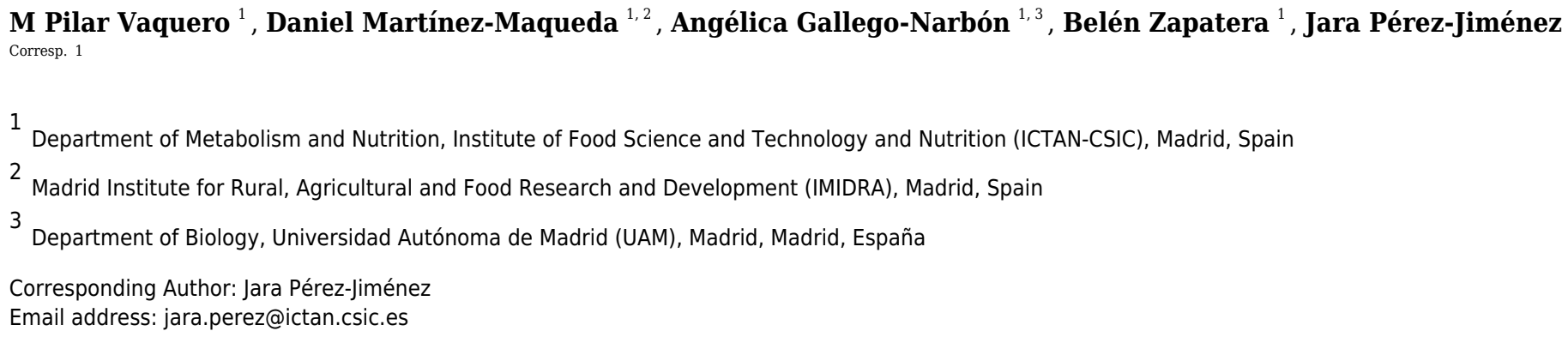

Background. Controversy exists on the relationship between iron metabolism and cardiometabolic risk. The aim of this study was to determine if there is a link between dysmetabolic iron and cardiometabolic markers in subjects with excess body weight.

Methods. Cross-sectional study with fifty participants presenting overweight or obesity and at least another metabolic syndrome factor. Determinations: anthropometry, body composition, blood pressure, lipids, glucose, insulin, leptin, areas under the curve (AUC) for glucose and insulin after an oral glucose tolerance test, hs-C reactive protein (hs-CRP), blood count, ferritin, transferrin, transferrin saturation (TSAT), soluble transferrin receptor (sTfR). Gender-adjusted linear correlations and two independent sample t tests were used.

Results. Ferritin was positively correlated with insulin-AUC $(r=0.547, p=0.008)$ and TSAT was negatively correlated with waist-hip ratio ( $r=-0.385, p=0.008)$, insulin $(r=-0.551, p<0.001)$, and insulin resistance (HOMA-IR, $r=-0.586, p<0.001)$. Subjects with TSAT $\leq 20 \%$ had higher insulin $(p=0.012)$ and HOMA-IR $(p=0.003)$ compared to those with TSAT $>20 \%$. In conclusion, the observed results suggest that iron transport and storage are altered in subjects with overweight/obesity, at the same time that they exhibit the characteristic features of insulin resistance. Nevertheless, this occurs without iron overload or deficiency. These results should be validated in wider cohorts since they suggest that iron transport and storage should be assessed when performing the clinical evaluation of subjects with excess body weight. 
1 Relationship between iron status markers and insulin resistance: an

2 exploratory study in subjects with excess body weight

3

4

5

6

7

8

9

10

11

12

13

14

15

16

17

18

19

20

21
M. Pilar Vaquero ${ }^{1}$, Daniel Martínez-Maqueda ${ }^{1,2}$, Angélica Gallego-Narbón ${ }^{1,3}$, Belén Zapatera ${ }^{1}$, Jara Pérez-Jiménez ${ }^{1}$

${ }^{1}$ Department of Metabolism and Nutrition, Institute of Food Science, Technology and Nutrition (ICTAN-CSIC), Madrid, Spain.

${ }^{2}$ Current affiliation: Madrid Institute for Rural, Agricultural and Food Research and Development (IMIDRA), Madrid, Spain.

${ }^{3}$ Current affiliation: Department of Biology, Universidad Autónoma de Madrid (UAM), Madrid, Spain

Corresponding Author:

Jara Pérez-Jiménez ${ }^{1}$

Department of Metabolism and Nutrition, Institute of Food Science, Technology and Nutrition (ICTAN-CSIC), José Antonio Novais 10, 28040, Madrid, Spain. Tel.: (+34) 915492300.

Email address: jara.perez@ictan.csic.es 


\section{Abstract}

23

27

Background. Controversy exists on the relationship between iron metabolism and cardiometabolic risk. The aim of this study was to determine if there is a link between dysmetabolic iron and cardiometabolic markers in subjects with excess body weight.

Methods. Cross-sectional study with fifty participants presenting overweight or obesity and at least another metabolic syndrome factor. Determinations: anthropometry, body composition, blood pressure, lipids, glucose, insulin, leptin, areas under the curve (AUC) for glucose and insulin after an oral glucose tolerance test, hs-C reactive protein (hs-CRP), blood count, ferritin, transferrin, transferrin saturation (TSAT), soluble transferrin receptor (sTfR). Gender-adjusted linear correlations and two independent sample $t$ tests were used.

Results. Ferritin was positively correlated with insulin-AUC $(r=0.547, p=0.008)$ and TSAT was negatively correlated with waist-hip ratio $(\mathrm{r}=-0.385, \mathrm{p}=0.008)$, insulin $(\mathrm{r}=-0.551, \mathrm{p}<0.001)$, and insulin resistance (HOMA-IR, $r=-0.586, p<0.001$ ). Subjects with TSAT $\leq 20 \%$ had higher insulin $(\mathrm{p}=0.012)$ and HOMA-IR $(\mathrm{p}=0.003)$ compared to those with TSAT $>20 \%$. In conclusion, the observed results suggest that iron transport and storage are altered in subjects with overweight/obesity, at the same time that they exhibit the characteristic features of insulin resistance. Nevertheless, this occurs without iron overload or deficiency. These results should be validated in wider cohorts since they suggest that iron transport and storage should be assessed when performing the clinical evaluation of subjects with excess body weight. 


\section{Introduction}

45

46

47

48

49

50

51

52

53

54

55

56

57

58

59

60

61

62

63

64

65

66

67

68

69

70

71

72

73

74

75

76

77

78

79

80

81

82

83

The worldwide epidemic of obesity that affects both industrial and developing countries (1) clearly has a multifactorial origin, therefore requiring a multidisciplinary approach. Overweight and obesity are the result of an imbalance between energy intake and expenditure, but they also involve a complex phenomenon of endocrine disruption signals. Obesity is one of the defining factors of metabolic syndrome (MetS), which is characterised by a cluster of factors also including excessive abdominal fat, dyslipaemia, hypertension, and insulin resistance (2).

Within this context, different studies have detected iron deficiency in obesity; this has been mainly attributed to the inflammatory status characteristic of excessive adipose tissue involving an elevation of proinflammatory cytokines such as hepcidin, which reduces iron absorption (3-5). In contrast, other reports show moderate to high levels of body iron in patients with cardiovascular diseases and type 2 diabetes (T2DM) (6), as well as associations between high ferritin and high triglycerides with low HDL-cholesterol in T2DM and patients with type 1 diabetes (7). Indeed, since iron activates many oxidative processes, excessive free iron may contribute either to the onset or to the aggravation of these pathologies. Therefore, if excess of iron is a contributing factor to cardiometabolic alterations, a reduction of its body levels should be beneficial. In this line, a reduction of body iron stores induced by phlebotomies decreased blood pressure and markers of cardiovascular risk, also improving glycaemic control, in patients with MetS (8). However, in patients with iron overload the benefit of this procedure compared to dietary and lifestyle intervention has not been proved (9). On the contrary, if iron deficiency coexists with obesity and contributes to fatigue, low physical performance, etc., iron supplementation should be promoted, but this has not been demonstrated and the issue is controversial. Another important aspect is that iron is a very well-known factor that increases food intake, which may increase body weight and adiposity in predisposed individuals, and, in this regard, one study suggested a relationship between adipocyte iron and appetite mediated by leptin, the satiety hormone (9).

Therefore, the complex metabolic alterations implicated in the MetS, with obesity as a key initial factor, may include dysmetabolic iron, although the specific direction of this alteration -excess or deficit- is not completely known and the role of iron in subjects with MetS, obesity, or diabetes is still limited and not conclusive.

Thus, the aim of this exploratory study was to evaluate the relationship between iron status parameters and both cardiometabolic risk factors as insulin resistance indexes in adults with overweight or obesity in order to advance in the knowledge of iron involvement in cardiometabolic alterations.

\section{Materials \& Methods}

PeerJ reviewing PDF | (2019:12:44300:2:1:NEW 19 Jun 2020) 
84

85

86

87

88

89

90

91

92

93

94

95

96

97

98

99

100

101

102

103

104

105

106

107

108

109

110

111

112

113

114

115

116

117

118

119

120

121

122

123

\section{Subjects and determinations}

This human study was conducted according to the Declaration of Helsinki guidelines. The participants were recruited in Madrid (Spain) and signed a written informed consent approved by the Ethics Committees of Hospital Puerta de Hierro-Majadahonda (2016/12/02) and the Spanish CSIC (2016/12/13). The design is a cross-sectional analytical study, part of the registered clinical trial NCT03076463. Inclusion criteria were: age $\geq 18$ and $<70$ years, apparently healthy and presenting body mass index (BMI) $>25 \mathrm{Kg} / \mathrm{m}^{2}$ and at least another MetS factor (fasting glucose $\geq$ $100 \mathrm{mg} \mathrm{dL}^{-1}$; HDL-cholesterol $\leq 50 \mathrm{mg} / \mathrm{dL}$ in women and $40 \mathrm{mg} / \mathrm{dL}$ in men; triglycerides $\geq 150$ $\mathrm{mg} / \mathrm{dL}$; systolic pressure $\geq 130 \mathrm{mmHg}$ or diastolic pressure $\geq 85 \mathrm{mmHg}$ ). Exclusion criteria were: diagnosis of any cardiometabolic pathologies, e.g., T2D; any previous cardiometabolic event, e.g., heart stroke; any medication related to cardiometabolic pathologies or risk factors, e.g., statins or angiotensin-converting enzyme inhibitors; any medication that may affect lipid profile; pregnancy; lactation.

Sample size was calculated using the $\mathrm{G}^{*}$ Power software for bivariate normal models. The primary outcome variable for sample size calculation was the modification in the HOMA-IR (homeostatic model assessment for insulin resistance) index. Based on previous studies $(7,11)$, assuming a coefficient $r=0.40, \alpha=0.05$ and statistical power $(1-\beta)=0.80$, a sample size of 46 was needed. Fifty subjects were recruited, and finally 49 subjects participated. Volunteers attended the Human Nutrition Unit of our institute for fasting analyses and, due to logistic reasons, an oral glucose tolerance test (OGTT) was performed on 25 randomly selected subjects.

Blood pressure, body weight, height, waist-hip ratio (WHR), and waist to height ratio (WHtR) were measured by standardized procedures and BMI was calculated. Body composition was estimated by bioimpedance (Tanita BC-601, Arlington heights, IL, USA). Blood samples were collected to determine: haematocrit, mean corpuscular volume (MCV), haemoglobin, red blood cell distribution width (RDW), serum iron, serum transferrin, glucose, lipids, high sensitive Creactive protein (hs-CRP), and serum ferritin, all by autoanalyzer. Serum soluble transferrin receptor (sTfR) was determined by ELISA (DRG Instruments GmbH, Marburg, Germany).

Food intake was assessed by three $24 \mathrm{~h}$ dietary recalls performed by telephone interviews (two in working days and one in the weekend) several days before blood sampling. They were performed by a dietitian, for about 10 minutes, the morning after the day that was registered. In order not to originate a modification in dietary habits, the subjects did not know in advance when they would receive the call. No diet record diaries had to be filled by volunteers. Daily energy intake, nutrient intake and energy provided by macronutrients were calculated with the computer program DIAL (Alce Ingeniería) using the Spanish Food Composition Database (12). 
124 An oral glucose tolerance test (OGTT) using a $75 \mathrm{~g}$ glucose solution was performed in a subsample 125 of 25 volunteers randomly selected. Serum samples were collected at 0, 30, 60 and $120 \mathrm{~min}$. 126 Glucose was analysed by the Free Style Optimum Neo blood glucose meter (Abbott, Chicago, IL, 127 USA) and insulin and leptin by ELISA assays (Merck-Millipore, Burlington, MS, USA). In the 128 case of the insulin kit (EZHI-14K), the standard curve range was 2-200 $\mu \mathrm{U} / \mathrm{mL}$ and the limit of 129 detection was $1 \mu \mathrm{U} / \mathrm{mL}$. In the case of the leptin kit (EZHL-80SK), the standard curve range was $0.5-100 \mathrm{ng} / \mathrm{mL}$ and the limit of detection was $0.2 \mathrm{ng} / \mathrm{mL}$.

131

132

\section{Calculations}

133

134

Total iron binding capacity $(\mathrm{TIBC})=25.1 \times \operatorname{transferrin}(\mathrm{g} / \mathrm{L})$.

136

137

138

Transferrin saturation $(\mathrm{TSAT})=\operatorname{serum}$ iron $(\mu \mathrm{mol} / \mathrm{L}) / \mathrm{TIBC}(\mu \mathrm{mol} / \mathrm{L}) \times 100$.

140

141

142

143

144

Insulin resistance was estimated by HOMA-IR $=($ glucose, $\mathrm{mg} / \mathrm{dL} \mathrm{x}$ insulin, $\mu \mathrm{U} / \mathrm{mL}) / 405$.

Areas under the curve (AUC) of glucose and insulin were calculated from the values obtained at $0,30,60$ and $120 \mathrm{~min}$ of the OGTT test using the trapezoid method.

\section{Statistical analyses}

The distribution of the variables was tested by the Shapiro-Wilk test. The relationship between variables was firstly explored by partial Pearson's correlations controlled by gender, using log- or square root-transformed variables when necessary, and p-values $\leq 0.01$ were considered significant. Two samples t test, or Mann-Whitney test for non-normally distributed variables, were used to compare groups of subjects classified according to gender and the TSAT cut-off value of $20 \%$ indicative of iron deficiency (13) and differences with $p<0.05$ were considered significant. Statistical analyses were performed with SPSS 24 (SPSS Inc., Chicago).

153

\section{Results}

All volunteers presented overweight or obesity, 65\% high blood pressure, $10 \%$ high glucose and $22 \%$ showed both MetS factors, without significant differences between genders. WHR, haematocrit, ferritin, sTfR, and the hepatic enzymes were significantly higher in men, while body fat, abdominal fat, transferrin, leptin and HDL-cholesterol were significantly higher in women (Table 1). Concerning dietary intake (mean of the three dietary recalls), men ingested significantly more energy, protein, lipids, cholesterol and calcium than women (Table 2), although values were 161 rather low for overweight/obese individuals.

Based on these cardiometabolic parameters, several correlations were assessed. BMI was positively correlated with body fat $(\mathrm{r}=0.859, \mathrm{p}<0.001)$, abdominal fat $(\mathrm{r}=0.804, \mathrm{p}<0.001)$, and 
164 HOMA-IR $(\mathrm{r}=0.395, \mathrm{p}<0.006)$. Transferrin and TSAT were negatively correlated $(\mathrm{r}=-0.450$,

165

166

167

168

169

170

171

172

173

174

175

176

177

178

179

180

181

182

183

184

185

186

187

188

189

190

191

192

193

194

195

196

197

198

199

200

201

202

203

$\mathrm{p}=0.002$ ) but there were no correlations between ferritin and TSAT, ferritin and sTfR, or transferrin and sTfR. In addition, ferritin was positively correlated with insulin-AUC $(r=0.547, p=0.008)$ while TSAT was negatively associated with WHR $(\mathrm{r}=-0.385, \mathrm{p}=0.008)$, insulin $(\mathrm{r}=-0.551$, $\mathrm{p}<0.001)$, and HOMA-IR $(\mathrm{r}=-0.586, \mathrm{p}<0.001)$. The relationship between ferritin and hs-CRP or between other iron and cardiometabolic markers did not reach the significance level $(p>0.01)$.

Additionally, the differences in cardiometabolic markers between subjects classified according to the TSAT cut-off value of $20 \%$ were evaluated (Table 3 ). Subjects in the low TSAT group (mean $\pm \mathrm{SD}, 15.8 \pm 3.4 \%$ ) presented significantly lower serum iron and significantly higher RDW, transferrin, TIBC, insulin, and HOMA-IR, and marginally higher hs-CRP. The differences in HOMA-IR between these groups remained when men and women were studied separately.

\section{Discussion}

In this exploratory study, the relationship between iron metabolism and cardiometabolic markers was evaluated in adults with overweight or obesity presenting at least another MetS factor. Results, obtained for the whole group as well as for the two groups classified according to the TSAT cutoff point, show a link between insulin resistance and iron storage, with low iron transport efficiency.

There is an intense debate on the possibility that iron dysregulation may be involved in insulin resistance. Population studies have reported associations between ferritin and cardiovascular markers, concluding that high body iron may be implicated in cardiovascular and diabetes risk (14, 15). However, in most cases ferritin values were in normal physiological range, what limits the relevance of the findings. Similarly, we did not observe either abnormally high or low ferritin values in this study on subjects with overweight/obesity. We found no association between this marker and body weight, in contrast with others $(3,4)$. Nevertheless, since ferritin is an acute phaseprotein, it was relevant to determine whether inflammation was a confounder factor. This was done by analysing hs-CRP, but the obtained values were below the $<2 \mathrm{mg} / \mathrm{dl}$ cut-off suggested for normality (16), so no association between ferritin and hs-CRP values was observed. Therefore, our results do not support that ferritin values were increased by inflammation in these individuals with overweight/obesity, which is in agreement with other studies (17). Moreover, the values of sTfR, that are unaffected by inflammation and reflect tissue iron needs, were about 50\% lower than those obtained in young iron-deficient women using the same analytical technique $(18,19)$, which denotes iron sufficiency in the present work.

The 20\% TSAT cut-off value has been proposed to detect iron deficiency (14). In the present study, the higher insulin and HOMA-IR values observed in the subjects with TSAT $<20 \%$ are in agreement with some results of the EPIC-InterAct prospective study (20), the KORA F4 study

PeerJ reviewing PDF | (2019:12:44300:2:1:NEW 19 Jun 2020) 
204 (21), and the US NHANES (14), all of them showing associations between low TSAT and 205 prediabetes. Another study reported that both low and high TSAT were associated with diabetes 206 risk (22). Interestingly, TSAT was moderately low in all volunteers and particularly in those of the 207 low TSAT group, meaning that iron binding to transferrin was insufficient (23). This group also 208 presented slightly higher hs-CRP than the other, although inflammation appears to be low. 209 Therefore, subjects with overweight/obesity may present insufficient delivery of iron to tissues, 210 which is related to insulin resistance and probably to a state of subclinical inflammation.

211

212 Different proteins play crucial roles in iron metabolism and are essential to guarantee iron binding 213 for transport and storage, acting as scavengers. In this regard, transferrin may be partly glycated 214 (24) and unable to efficiently bind and release iron, with possible physiological consequences. 215 Since labile iron acts as a powerful pro-oxidant, free non-transferrin-bound iron may involve 216 oxidative stress, which in turn would increase cardiovascular and T2DM risks $(6,25)$. Indeed, an 217 elevation of non-transferrin-bound iron has been observed in patients with T2DM (26). This is 218 consistent with the condition of insulin resistance observed in the present study. Leptin was also 219 elevated in these subjects with overweight/obesity: although no reference values have been 220 internationally established, the concentrations obtained here were above the cut-off values for 221 cardiometabolic abnormalities reported in a Spanish sample of 11,000 subjects, i.e, $6.45 \mathrm{ng} / \mathrm{ml}$ for 222 males and $23.75 \mathrm{ng} / \mathrm{ml}$ for females (27). Nevertheless, this increase in leptin concentration was not 223 related to any iron marker value. Therefore, considering the haematological, transferrin, TSAT, 224 sTfR and ferritin results, it can be concluded that iron status was enough for haemoglobin 225 maintenance, but transferrin was inefficient. Moreover, these iron metabolism proteins appear to 226 reflect a situation of altered iron handling and iron displacement.

227

228

229

230

231

232

233

234

\section{Conclusions}

236

237 Characteristics of iron deficiency or iron overload were not observed in subjects with 238 overweight/obesity exhibiting at least one additional MetS factor. The issue of transferrin and its 239 binding capacity implication on insulin resistance is much more controversial than that of ferritin, 240 and our results suggest a complex metabolic dysregulation. Priority research should be focused 241 not only on answering the question of whether body iron is increased or decreased in MetS, obesity 242 and T2DM, but rather if dysmetabolic iron occurs with delocalization of iron, thus increasing its 243 free radical potential and health consequences. 
244

245

246

247

248

249

250

251

252

253

254

255

256

257

258

259

260

261

262

263

264

265

266

267

268

269

270

271

272

273

274

275

276

277

278

279

280

281

282

\section{Acknowledgements}

Volunteers are kindly acknowledged for their participation in the study.

\section{References}

1. NCD Risk Factor Collaboration (NCD-RisC). Trends in adult body-mass index in 200 countries from 1975 to 2014: a pooled analysis of 1698 population-based measurement studies with 19.2 million participants. Lancet 2016; 387:1377-1396.

2. Alberti KG, Eckel RH, Grundy SM, Zimmet PZ, Cleeman JI, Donato KA, Fruchart JC, James WP, Loria CM, Smith SC, Jr. Harmonizing the metabolic syndrome: a joint interim statement of the International Diabetes Federation Task Force on Epidemiology and Prevention; National Heart, Lung, and Blood Institute; American Heart Association; World Heart Federation; International Atherosclerosis Society; and International Association for the Study of Obesity. Circulation 2009; 120:1640-1645.

3. Tussing-Humphreys LM, Liang H, Nemeth E, Freels S, Braunschweig CA. Excess adiposity, inflammation, and iron-deficiency in female adolescents. J Am Diet Assoc 2009; 109: 297-302.

4. Aigner E, Feldman A, Datz C. Obesity as an emerging risk factor for iron deficiency. Nutrients 2014; 6: 3587-3600.

5. Zhao L, Zhang X, Shen Y, Fang X, Wang Y, Wang F. Obesity and iron deficiency: a quantitative meta-analysis. Obesity Rev 2015; 16: 1081-1093.

6. Zacharski LR, Shamayeva G, Chow BK, DePalma RG. Ferritin and percent transferrin saturation levels predict type 2 diabetes risk and cardiovascular disease outcomes. Curr Diabetes Rev 2017; 13: 428-436.

7. Vaquero MP, García-Quismondo Á, Del Cañizo FJ, Sánchez-Muniz FJ. Iron status biomarkers and cardiovascular risk. In: Kumar A, ed. Recent Trends in Cardiovascular Risks. London: IntechOpen; 2017. pp. 97-117.

8. Houschyar KS, Ludtke R, Dobos GJ, Kalus U, Broecker-Preuss M, Rampp T, Brinkhaus B, Michalsen A. Effects of phlebotomy-induced reduction of body iron stores on metabolic syndrome: results from a randomized clinical trial. BMC Medicine 2012; 10:54.

9. Laine F, Ruivard M, Loustaud-Ratti V, Bonnett F, Calès P, Bardou-Jacquet E, SacherHuvelin S, Causse X, Beusnel C, Renault A, Bellissant E, Deugnier Y. Metabolic and hepatic effects of bloodletting in dysmetabolic iron overload syndrome: A randomized controlled study in 274 patients. Hepatology 2017; 65: 465-474.

10. Gao Y, Li Z, Gabrielsen JS, Simcox JA, Lee SH, Jones D, Cooksey B, Stoddard G, Cefalu WT, McClain DA (2015) Adipocyte iron regulates leptin and food intake. J Clin Invest 2015; 125:3681-3691.

11. Ebron K, Andersen CJ, Aguilar D, Blesso CN, Barona J, Dugan CE, Jones JL, Al-Sarraj $\mathrm{T}$, Fernandez ML. A larger body mass index is associated with increased atherogenic dyslipidemia, 
283 insulin resistance, and low-grade inflammation in individuals with metabolic syndrome. Metab 284 Syndr Relat Disord 2015; 13:458-464.

285 12. Ortega RM, López-Sobaler AM, Andrés P, Requejo AM, Aparicio A, Molinero LM. DIAL 286 1.0, Programa DIAL para valoración de dietas y cálculos de alimentación. Departamento de 287 Nutrición (UCM) y Alceingeniería, 2013, Madrid, Spain.

288 13. Wish JB. Assessing iron status: beyond serum ferritin and transferrin saturation. Clin J Am 289 Soc Nephrol 2006; 1 Suppl 1: S4-S8.

290 14. Cheung CL, Cheung TT, Lam KS, Cheung BM. High ferritin and low transferrin saturation 291 are associated with pre-diabetes among a national representative sample of U.S. adults. Clin Nutr 292 2013; 32: 1055-1060.

293 15. Wlazlo N, van Greevenbroek MM, Ferreira I, Jansen EH, Feskens EJ, van der Kallen CJ, 294 Schalkwijk CG, Bravenboer B, Stehouwer CD. Iron metabolism is prospectively associated with 295 insulin resistance and glucose intolerance over a 7-year follow-up period: the CODAM study. Acta 296 Diabetol 2015; 52: 337-348.

297 16. Goff DC, Jr., Lloyd-Jones DM, Bennett G, Coady S, D’Agostino RB, Gibbons R, 298 Greenland P, Lackland DT, Levy D, O’Donnell CJ, Robinson JG, Schwartz JS, Shero ST, Smith 299 SC, Sorlie P, Stone NJ, Wilson PWF. 2013 ACC/AHA guideline on the assessment of 300 cardiovascular risk: a report of the American College of Cardiology/American Heart Association 301 Task Force on Practice Guidelines. J Am Coll Cardiol 2014; 63: 2935-2959.

302 17. Crist BL, Alekel DL, Ritland LM, Hanson LN, Genschel U, Reddy MB. Association of 303 oxidative stress, iron, and centralized fat mass in healthy postmenopausal women. J Women's 304 Health (Larchmt) 2009; 18: 795-801.

305 22. Blanco-Rojo R, Perez-Granados AM, Toxqui L, Gonzalez-Vizcayno C, Delgado MA, 306 Vaquero MP. Efficacy of a microencapsulated iron pyrophosphate-fortified fruit juice: a 307 randomised, double-blind, placebo-controlled study in Spanish iron-deficient women. Br J Nutr 308 2011; 105: 1652-1659.

309 19. Toxqui L, Perez-Granados AM, Blanco-Rojo R, Wright I, Gonzalez-Vizcayno C, Vaquero 310 MP. Effects of an iron or iron and vitamin D-fortified flavored skim milk on iron metabolism: a 311 randomized controlled double-blind trial in iron-deficient women. J Am Coll Nutr 2013; 32: 312312320.

313 20. Podmore C, Meidtner K, Schulze MB, Scott RA, Ramond A, Butterworth AS, 314 DiAngelantonio E, Danesh J, Arriola L, Barricarte A, Boeing H, Clavel-Chapelon F, Cross AJ, 315 Dahm CC, Fagherazzi G, Franks PW, Gavrila D, Grioni S, Gunter MJ, Gusto G, Jakszyn P, Katzke 316 V, Key TJ, Kühn T, Mattiello A, Nilsson PM, Olsen A, Overvad K, Palli D, Quirós JR, Rolandsson 317 O, Sacerdote C, Sánchez-Cantalejo E, Slimani E, Sluijs I, Spijkerman AM, Tjonneland A, Tumino 318 R, Van der A DL, Van der Schouw YT, Feskens EJ, Forouhi NG, Sharp SJ, Riboli E, Langenberg 319 C, Wareham NJ . Association of multiple biomarkers of iron metabolism and type 2 diabetes: The 320 EPIC-InterAct Study. Diabetes Care 2016; 39: 572-581.

321 21. Huth C, Beuerle S, Zierer A, Heier M, Herder C, Kaiser T, Koenig W, Kronenberg F, Oexle 322 K, Rathmann W, Roden M, Schwab S, Seissler J, Stöckl D, Meisinger C, Peters A, Thorand B. 
323 Biomarkers of iron metabolism are independently associated with impaired glucose metabolism 324 and type 2 diabetes: the KORA F4 study. Eur J Endocrinol 2015; 173: 643-653.

325 22. Ellervik C, Mandrup-Poulsen T, Andersen HU, Tybjaerg-Hansen A, Frandsen M, Birgens 326 H, Nordestgaard BG. Transferrin saturation and risk of diabetes. Diabetes Care 2011; 34: 2256.

327 23. Stack AG, Mutwali AI, Nguyen HT, Cronin CJ, Casserly LF, Ferguson J. Transferrin 328 saturation ratio and risk of total and cardiovascular mortality in the general population. QJM 2014; 329 107: 623-633.

330 24. Fernandez-Real JM, McClain D, Manco M. Mechanisms linking glucose homeostasis and 331 iron metabolism toward the onset and progression of type 2 diabetes. Diabetes Care 2015; 38: 332 2169-2176.

333 25. Montonen J, Boeing H, Steffen A, Lehmann R, Fritsche A, Joost HG, Schulze MB, Pischon 334 T. Body iron stores and risk of type 2 diabetes: results from the European Prospective Investigation 335 into Cancer and Nutrition (EPIC)-Potsdam study. Diabetologia 2012; 55: 2613-2621.

336 26. Lee DH, Liu DY, Jacobs DR, Shin HR, Song K, Lee IK, Kim B, Hider RC. Common 337 presence of non-transferrin-bound iron among patients with type 2 diabetes. Diabetes Care 2006; 338 29: 1090-1095.

339 27. Gijón-Conde T, Graciani A, Guallar-Castillón, Aguilera MT, Rodríguez-Artalejo F, 340 Banegas JR . Leptin reference values and cutoffs for identifying cardiometabolic abnormalities in 341 the Spanish population. Revista Española de Cardiología 2015; 68: 672-79. 


\section{Table $\mathbf{1}$ (on next page)}

Table 1

Body composition, blood pressure, haematological, and biochemical markers of the participants. 
1 TABLE 1. Body composition, blood pressure, haematological, and biochemical markers of the 2 participants.
Men
Women
$P$ value

\section{General characteristics}

Number of subjects

Age (years)

$\operatorname{BMI}\left(\mathrm{Kg} / \mathrm{m}^{2}\right)$

WHR

WHtR

Total body fat (\%)

Abdominal fat (\%)

Systolic pressure

(mmHg)

Diastolic pressure

(mmHg)

Haematological parameters

Haemoglobin (g/dl)

Haematocrit (\%)

MCV (fL)

RDW (\%)

Iron $(\mu \mathrm{mol} / 1)$

Ferritin (ng/ml)

Transferrin (g/l)

TSAT (\%)

TIBC $(\mu \mathrm{mol} / 1)$

27
$42.5 \pm 10.8$

$29.8(32.0 ; 27.9)$

$0.99 \pm 0.05$

$0.60 \pm 0.07$

$27.0 \pm 6.0$

$29.3 \pm 6.5$

$120.8 \pm 11.1$

$83.2 \pm 7.8$

$14.0 \pm 0.9$

$<0.001$

$47.0(48.2 ; 45.5)$

$90.0 \pm 3.8$

$13.5 \pm 0.9$

$15.0 \pm 5.1$

$160(224.5 ; 89.0)$

$2.8 \pm 0.3$

$22.8 \pm 5.3$

$70.3 \pm 7.5$

$43.0(44.4 ; 40.4)$

$<0.001$

$90.8 \pm 5.9$

0.593

$13.2 \pm 0.6$

0.169

$16.6 \pm 6.2$

0.328

$<0.001$

0.014

$$
3.1 \pm 0.4
$$

0.883

$23.0 \pm 7.4$

0.014

$76.8 \pm 9.9$

$\operatorname{sTfR}(\mu \mathrm{g} / \mathrm{ml})$

$0.99(1.15 ; 0.88)$

0.015

Cardiometabolic markers

Glucose (mg/dl)

$99.1 \pm 9.1$

$98.0 \pm 12.0$

0.735

Insulin $(\mu \mathrm{U} / \mathrm{ml})$
$7.0 \pm 2.3$
$7.4 \pm 3.7$

0.635 


\begin{tabular}{lccc}
\hline Leptin $(\mathrm{ng} / \mathrm{ml})$ & $13.5(28.9 ; 7.2)$ & $33.6(48.6 ; 26.2)$ & $\mathbf{0 . 0 0 2}$ \\
Total cholesterol $(\mathrm{mg} / \mathrm{dl})$ & $199.1 \pm 31.9$ & $208.6 \pm 53.5$ & 0.442 \\
LDL-cholesterol $(\mathrm{mg} / \mathrm{dl})$ & $123.4 \pm 30.0$ & $122.3 \pm 30.0$ & 0.898 \\
HDL-cholesterol $(\mathrm{mg} / \mathrm{dl})$ & $44.1 \pm 9.6$ & $53.8 \pm 11.0$ & $\mathbf{0 . 0 0 2}$ \\
Triglycerides $(\mathrm{mg} / \mathrm{dl})$ & $135.5(210.5 ; 106)$ & $119.5(156.5 ; 85.0)$ & 0.183 \\
hs-CRP $(\mathrm{mg} / \mathrm{L})$ & $1.34(3.55 ; 0.62)$ & $3.32(6.60 ; 0.70)$ & 0.117 \\
HOMA-IR & $1.62(2.07 ; 1.36)$ & $1.58(2.11 ; 1.15)$ & 0.355 \\
\hline
\end{tabular}

3 Values are shown as mean \pm SD for normal variables, and as median (percentile 75;percentile 25)

4 for non-normal variables. BMI, Body Mass Index; WHR, Waist to Hip Ratio; WHtR, Waist to 5 Height Ratio; MCV, Mean Corpuscular Volume; RDW, Red blood cell Distribution Width; TSAT,

6 Transferrin Saturation; TIBC, Total Iron Binding Capacity; sTfR, Soluble Serum Transferrin 7 Receptor; hs-CRP, high sensitivity-C Reactive Protein; HOMA-IR, Homeostasis Model 8 Assessment Index. 
Table 2 (on next page)

Table 2

Diet characteristics of the participants of the study. 
1 Table 2. Diet characteristics of the participants of the study.

\begin{tabular}{|c|c|c|c|}
\hline & Men & Women & Pvalue ${ }^{\mathrm{a}}$ \\
\hline Energy (Kcal) & $2055 \pm 565$ & $1674 \pm 620$ & 0.037 \\
\hline Protein (g) & $89.4 \pm 17.9$ & $68.3 \pm 26.3$ & 0.003 \\
\hline Carbohydrates (g) & $196.4 \pm 58.8$ & $178.0 \pm 77.0$ & 0.368 \\
\hline Fibre (g) & $21.7(32.4 ; 16.0)$ & $18.3(29.1 ; 14.8)$ & 0.328 \\
\hline Lipids (g) & $91.4 \pm 32.3$ & $69.4 \pm 30.6$ & 0.024 \\
\hline Cholesterol (mg) & $319.6 \pm 101.7$ & $253.7 \pm 114.8$ & 0.047 \\
\hline $\mathrm{SFA}^{\mathrm{b}}(\%$ energy $)$ & $13.0 \pm 2.7$ & $12.5 \pm 2.8$ & 0.568 \\
\hline MUFA $^{c}(\%$ energy) & $16.8 \pm 4.3$ & $14.7 \pm 5.0$ & 0.159 \\
\hline PUFA $^{d}$ (\% energy) & $5.8 \pm 1.9$ & $5.9 \pm 1.6$ & 0.811 \\
\hline Calcium (mg) & $906.0 \pm 340.0$ & $625.5 \pm 262.4$ & 0.004 \\
\hline Iron (mg) & $14.8 \pm 4.7$ & $12.5 \pm 4.7$ & 0.105 \\
\hline Vitamin A $(\mu g)$ & $683(1210 ; 573)$ & $568(797 ; 424)$ & 0.133 \\
\hline Vitamin $B_{1}(\mathrm{mg})$ & $1.3(1.9 ; 1.1)$ & $1.2(1.7 ; 1.0)$ & 0.315 \\
\hline Vitamin $B_{2}(m g)$ & $1.9 \pm 0.6$ & $1.5 \pm 0.6$ & 0.068 \\
\hline Folic acid $(\mu \mathrm{g})$ & $278(343 ; 193)$ & $259(286 ; 172)$ & 0.307 \\
\hline Vitamin C (mg) & $117(201 ; 55)$ & $107(157 ; 73)$ & 0.672 \\
\hline
\end{tabular}

$2{ }^{a}$ Mean values for 3 days dietary recalls. Values are expressed as mean \pm SD for normal variables,

3 and as median (percentile 75; percentile 25) for non-normal variables.

$4 \quad$ bFA $=$ Saturated Fatty acids.

$5 \quad$ MUFA=Monounsaturated Fatty Acids. 
$6 \quad$ dPUFA=Polyunsaturated Fatty Acids.

7

Peer] reviewing PDF | (2019:12:44300:2:1:NEW 19 Jun 2020) 


\section{Table 3(on next page)}

Table 3

Body composition, blood pressure, biochemical markers and cardiometabolic indexes according to transferrin saturation classification 
1 TABLE 3. Body composition, blood pressure, biochemical markers and cardiometabolic indexes

2 according to transferrin saturation classification

\begin{tabular}{|c|c|c|c|}
\hline & $\begin{array}{c}\text { Group } 1 \\
\text { TSAT } \leq 20 \%\end{array}$ & Group 2 TSAT $>20 \%$ & Pvalue \\
\hline \multicolumn{4}{|l|}{ General characteristics } \\
\hline Women $(\%, n)$ & $50.0(8)$ & $42.4(14)$ & 0.789 \\
\hline Age (years) & $39.4 \pm 12.8$ & $44.2 \pm 10.9$ & 0.185 \\
\hline $\operatorname{BMI}\left(\mathrm{Kg} / \mathrm{m}^{2}\right)$ & $30.2(36.5 ; 26.0)$ & $29.5(32.2 ; 26.9)$ & 0.714 \\
\hline WHR & $0.95 \pm 0.06$ & $0.94 \pm 0.09$ & 0.429 \\
\hline WHtR & $0.62 \pm 0.08$ & $0.60 \pm 0.06$ & 0.467 \\
\hline Total body fat (\%) & $32.9 \pm 10.0$ & $32.1 \pm 8.6$ & 0.794 \\
\hline Abdominal fat $(\%)$ & $33.2 \pm 9.4$ & $32.2 \pm 8.0$ & 0.683 \\
\hline Systolic pressure (mmHg) & $116.8 \pm 10.1$ & $119.1 \pm 14.3$ & 0.577 \\
\hline Diastolic pressure (mmHg) & $82.4 \pm 8.0$ & $82.3 \pm 9.6$ & 0.964 \\
\hline \multicolumn{4}{|l|}{ Haematological parameters } \\
\hline Haemoglobin (g/dl) & $14.5 \pm 1.6$ & $15.2 \pm 1.1$ & 0.087 \\
\hline Haematocrit (\%) & $45.0(47.0 ; 39.9)$ & $45.5(47.7 ; 43.6)$ & 0.175 \\
\hline MCV (fL) & $89.5 \pm 4.7$ & $90.7 \pm 4.9$ & 0.442 \\
\hline RDW (\%) & $14.0 \pm 0.9$ & $13.1 \pm 0.5$ & 0.005 \\
\hline Iron $(\mu \mathrm{mol} / 1)$ & $10.8 \pm 4.5$ & $17.9 \pm 4.5$ & $<0.001$ \\
\hline Ferritin (ng/ml) & $36.4(166.5 ; 22.9)$ & $100.5(184.3 ; 63.6)$ & 0.072 \\
\hline Transferrin $(\mathrm{g} / \mathrm{l})$ & $3.1 \pm 0.4$ & $2.8 \pm 0.3$ & 0.006 \\
\hline TSAT $(\%)$ & $15.8 \pm 3.4$ & $26.1 \pm 4.3$ & $<0.001$ \\
\hline TIBC $(\mu \mathrm{mol} / 1)$ & $78.4 \pm 9.9$ & $70.8 \pm 7.8$ & 0.006 \\
\hline $\operatorname{sTfR}(\mu \mathrm{g} / \mathrm{ml})$ & $1.02(1.17 ; 0.74)$ & $0.93(1.0 ; 0.73)$ & 0.151 \\
\hline \multicolumn{4}{|l|}{ Cardiometabolic markers } \\
\hline Glucose (mg/dl) & $100.7 \pm 14.0$ & $97.5 \pm 8.2$ & 0.437 \\
\hline Insulin $(\mu \mathrm{U} / \mathrm{ml})$ & $8.9 \pm 3.7$ & $6.5 \pm 2.3$ & 0.012 \\
\hline Leptin (ng/ml) & $22.7(42.0 ; 11.2)$ & $22.5(33.1 ; 9.1)$ & 0.852 \\
\hline Total-cholesterol (mg/dl) & $208.2 \pm 55.7$ & $202.6 \pm 36.1$ & 0.678 \\
\hline
\end{tabular}




\begin{tabular}{lccc}
\hline LDL-cholesterol $(\mathrm{mg} / \mathrm{dl})$ & $120.4 \pm 27.6$ & $125.4 \pm 30.3$ & 0.593 \\
HDL-cholesterol $(\mathrm{mg} / \mathrm{dl})$ & $46.8 \pm 11.9$ & $48.9 \pm 11.1$ & 0.554 \\
Triglycerides $(\mathrm{mg} / \mathrm{dl})$ & $123.5(215.9 ; 115.1)$ & $135.5(182.0 ; 79.5)$ & 0.471 \\
hs-CRP (g/L) & $3.65(8.38 ; 8.14)$ & $1.72(3.33 ; 4.22)$ & 0.060 \\
HOMA-IR & $2.01(3.89 ; 1.54)$ & $1.42(1.88 ; 1.14)$ & $\mathbf{0 . 0 0 3}$
\end{tabular}

3 Values are mean \pm SD except for non-parametric variables which are expressed as median

4 (percentile 75; percentile 25). TSAT, Transferrin Saturation; BMI, Body Mass Index; WHR, Waist

5 to Hip Ratio; WHtR, Waist to Height Ratio; MCV, Mean Corpuscular Volume; RDW, Red blood

6 cell Distribution Width; TIBC, Total Iron Binding Capacity. sTfR, Soluble Serum Transferrin

7 Receptor; hs-CRP, high sensitivity-C Reactive Protein; HOMA-IR, Homeostasis Model

8 Assessment Index.

9 